OPEN ACCESS

Edited by:

Pedro Morgado,

University of Minho, Portugal

Reviewed by:

George P. Chrousos,

National and Kapodistrian University

of Athens, Greece

Amit Anand,

Case Western Reserve University,

United States

${ }^{*}$ Correspondence:

William Davies

daviesw4@cardiff.ac.uk

Specialty section:

This article was submitted to Mood and Anxiety Disorders,

a section of the journal

Frontiers in Psychiatry

Received: 18 July 2019 Accepted: 06 November 2019

Published: 26 November 2019

Citation:

Davies W (2019) An Analysis of Cellular

Communication Network Factor

Proteins as Candidate Mediators of

Postpartum Psychosis Risk.

Front. Psychiatry 10:876.

doi: 10.3389/fpsyt.2019.00876

\section{An Analysis of Cellular Communication Network Factor Proteins as Candidate Mediators of Postpartum Psychosis Risk}

\author{
William Davies ${ }^{1,2,3 *}$
}

${ }^{1}$ MRC Centre for Neuropsychiatric Genetics and Genomics and Division of Psychological Medicine and Clinical Neurosciences, School of Medicine, Cardiff University, Cardiff, United Kingdom, ${ }^{2}$ School of Psychology, Cardiff University, Cardiff, United Kingdom, ${ }^{3}$ Neuroscience and Mental Health Research Institute, Cardiff University, Cardiff, United Kingdom

Postpartum (or puerperal) psychosis (PP) is a severe psychiatric condition associated with hallucinations, delusions, cognitive disorganization, and mood problems, which affects approximately 1-2 out of every 1,000 mothers shortly after childbirth. While the risk factors for, and co-morbidities of, PP are relatively well-defined, currently, the pathophysiology underlying the disorder is very poorly-specified. Here, I argue, on the basis of multiple lines of new evidence, that altered expression of the Cellular Communication Network (CCN) factor proteins (and of the heterodimerizing CCN2 and CCN3 proteins in particular), may be associated with, and possibly causal for, increased PP risk. Future preclinical and clinical studies should aim to test this hypothesis as empirical support for it would provide much-needed clues regarding the biological substrates of PP, and could point to predictive biomarkers for the condition.

Keywords: brain, connective tissue growth factor, mood disorder, myelination, nephroblastoma-overexpressed

\section{POSTPARTUM PSYCHOSIS: AN INTRODUCTION}

Postpartum (or puerperal) psychosis (PP) is a severe psychiatric condition affecting approximately 1-2 of every 1,000 new mothers (1). The disorder is characterized by multiple symptoms occurring shortly after childbirth, often within the first few days; these can include hallucinations, delusions (often related to the baby), cognitive disorganization, and mood abnormalities including mania, depressive episodes, and extreme anxiety (2). PP can severely impact upon mother-child bonding and family dynamics, and is a leading cause of maternal death via suicide, and infanticide (3). Treatments for PP are relatively effective in many cases if administered promptly, and include a combination of antipsychotic, mood-stabilizing and sedative/anxiolytic drugs, and psychosocial support (2). However, some drugs lack efficacy, or exhibit unwanted side-effects, in some patients, and there are risks with administering some pharmacological treatments prophylactically during pregnancy due to teratogenicity issues (4). Thus, there remains a need for alternative treatments to be developed, which will, in turn, require an understanding of PP pathophysiology.

Our current understanding of PP molecular pathophysiology is extremely poor for a number of reasons, including the inaccessibility of the human brain, the low prevalence and high symptom heterogeneity of the condition, and a historic lack of relevant animal models (5). Clinical studies have tended to focus upon obvious candidate biological systems in the perinatal period, including variations in steroid hormone levels, immune system fluctuations, and genes influencing the 
dopaminergic, serotonergic, and stress-response systems. While there is some evidence for altered sensitivity to postpartum steroid hormone levels, immune system hyperactivation, and a nominally-increased frequency of specific genetic variants within serotonergic genes in patients with PP, results from clinical studies so far have been limited and inconsistent (5). The nature of PP limits our ability to perform robust, hypothesis-free, largescale biochemical, and genetic analyses, including genome-wide analyses which are now beginning to bear fruit in helping us to understand the biological basis of other, more common, mood, and psychotic illnesses (6); even with ongoing multi-national collaborative efforts, the sample sizes required to identify a reasonable proportion of genetic risk variants (and thereafter risk pathways) are unlikely to be achieved in the near future. Hence, for rare disorders such as $\mathrm{PP}$, investigations of sensible candidate biological pathways may still be warranted.

Ideally, we would like to know during pregnancy, or even before pregnancy, which women are at increased risk of being affected by PP, so that they can be closely monitored and receive early intervention for maximum therapeutic benefit. While we know that women with a previous history of PP, bipolar disorder or schizophrenia are at substantially increased risk of PP, approximately half of women who experience PP have no prior psychiatric history (7). Biological differences between women experiencing PP, and those who do not, could potentially represent predictive biomarkers for the condition.

In this perspective, I hypothesize, based upon several lines of converging preclinical and clinical evidence, that the expression of Cellular Communication Network (CCN) family member proteins may be disrupted in women at risk of $\mathrm{PP}$, and that detailed analysis of this system in future studies could feasibly yield insights into the pathophysiology of PP and signpost predictive biomarkers.

\section{THE CELLULAR COMMUNICATION NETWORK (CCN) FACTOR GENE FAMILY}

The CCN gene family comprises six members formerly known as cysteine-rich angiogenic inducer 61 (CYR61, now CCN1), connective tissue growth factor (CTGF/CCN2), nephroblastoma-overexpressed (NOV/CCN3), WNT1-inducible signaling pathway 1 (WISP1/CCN4), WNT1-inducible signaling pathway 2 (WISP2/CCN5), and WNT1-inducible signaling pathway 3 (WISP3/CCN6). The family member proteins share a tetramodular domain and regulate a variety of developmental and physiological processes including cell adhesion, migration, proliferation, differentiation, and survival (8). Although CCN proteins are expressed intracellularly, they appear to play a particularly important role within the extracellular matrix where they interact with multiple components, including a variety of cell membrane receptors (8); this site of action is pertinent as extracellular matrix proteins have been highlighted as candidate modulators of mood disorders (9). The CT domain shared across CCN members is a common motif in dimerizing proteins (10), and there is evidence that CCN members such as CCN2 and CCN3 can form homo or heterodimers; these two proteins may exert opposing effects (11), and in the nervous system, this is exemplified by CCN2 and CCN3's anti- and pro-myelination effects, respectively (12-14). Below, I argue that six key features of the CCN family member proteins (and specifically CCN2 and $\mathrm{CCN} 3$ ), render them plausible biological mediators of PP risk.

\section{Altered Gene Expression in Animal Models Displaying Abnormal Maternal Behavior}

It should initially be acknowledged that complex psychiatric conditions such as psychosis are challenging to model accurately or comprehensively in animals (15). This caveat notwithstanding, recent work in three independent, and distinct, animal models demonstrating abnormal maternal behavioural phenotypes has indicated correlated CCN2 and CCN3 brain gene expression changes.

In a pharmacological mouse model, the steroid sulfatase (STS) enzyme was acutely inhibited in new mothers to mimic postpartum steroid sulfatase deficiency which is associated with increased postpartum psychopathology risk (16) [including potentially increased postpartum psychosis risk (17)] in women. Mouse mothers in which STS was inhibited exhibited apparently normal dam-pup interactions, but altered anxietyrelated behaviors and a reduced startle response; the specific pattern of anxiety-related behaviors implicated an underlying genetic substrate within a small region of chromosome 15 (18). Screening of the 17 genes within this interval identified CCN3 as the only significantly differentially expressed (upregulated) gene in the brain between vehicle and inhibitor-treated mothers, and follow-up analyses of other CCN family members demonstrated significant brain overexpression of CCN2 and CCN4 genes in the latter group (18).

In a genetic mouse model, the postpartum behavior of wild-type mothers bearing transgenic pups overexpressing the imprinted gene Phlda2 was compared to that of wild-type mothers bearing wild-type pups (19). After birth, wild-type dams exposed in utero to transgenic offspring exhibited decreased nursing and grooming of pups, and an increased focus on nest building, relative to wild-type mothers exposed to wild-type pups. Analysis of global gene expression in the hippocampus and hypothalamus of mothers bearing transgenic pups revealed several significant differences from that in mothers bearing wildtype pups, including substantial upregulation of hippocampal Ccn 3 expression (>2.1-fold change) (19).

In pigs, a small proportion of new mothers demonstrate aggression towards their offspring, in some cases killing one or all of the litter. The "infantidal sow" was the first animal model proposed for PP, and exhibits some degree of face validity for the condition: the extent of aggression is influenced by familial, hormonal, and environmental factors, is greater for first litters, and is associated with behavioral correlates such as anxiety and restlessness (20). A preliminary analysis employing RNA sequencing to compare hypothalamic gene expression in infanticidal and non-infanticidal sows revealed increased CCN2 (CTGF) expression ( 1.3-fold) in the former group as one of the top 10 largest expression differences (21). Genetic analysis of the pig model has also implicated $P A X 3$ in postpartum aggression (22); the chromosomal 
translocation-derived fusion transcription factor PAX3-FKHR appears to act as a transcriptional activator at CCN3 (23).

\section{Location Under a Genetic Linkage Peak for PP}

A seminal genetic study in a population of women with bipolar disorder who developed PP identified a genome-wide significant linkage signal on chromosome $16 \mathrm{p} 13$, and a genome-wide suggestive linkage signal at 8q24 (24). The suggestive linkage signal spanned $14 \mathrm{~cm}$, from $135.7-149.7 \mathrm{~cm}$; the CCN3 gene is located at $138 \mathrm{~cm}$, and as such could potentially fully, or partially, explain this signal.

\section{PP-Relevant Spatiotemporal Gene Expression Patterns and Influence on Relevant Behavioral Phenotypes}

$\mathrm{CCN}$ genes are expressed in the developing and adult mammalian brain, with associated proteins apparently more highly expressed in neurons than in glia (25). Across species, the highest expression of CCN2 and CCN3 is seen in brain regions underpinning higher cognitive functions and emotionality, including the hippocampus (CA1 region), and frontal and temporal cortices (25-27); interestingly, these brain regions appear to be structurally distinct in women at risk of PP who develop the condition relative to those who don't (28), while alterations in the structure and function of the CA1 region of the hippocampus are among the most replicated neuroimaging findings in individuals with psychotic and/or bipolar illness (29).

A priori, one might expect the expression of candidate genes for PP to fluctuate within the mother's brain between late pregnancy and into the postpartum period. Although information on the brain expression of CCN genes across these timepoints in humans is inevitably lacking, there is some evidence from rodents that maternal brain CCN expression does change in a spatiotemporally-specific manner from mid-late gestation and into the postpartum period (30). Notably, CCN3 expression decreases in both the maternal neocortex and cerebellum across these timepoints, but increases significantly in the hypothalamus (Figure 1); CCN2 levels, in contrast, remain relatively stable across all four brain regions.

If there is a true link between abnormal CCN family gene expression and PP, we might reasonably expect to see altered expression of these genes/proteins in tissue samples from individuals with psychotic illness. In a recent comparison of global gene expression in prefrontal parvalbumin cells from individuals with schizophrenia versus healthy controls, more than 800 transcripts were identified as being differentially expressed, with CCN3 being one of the top hits ( $>40 \%$ change in expression) (31). Second, an analysis of plasma analytes in individuals at high risk of developing psychosis has suggested that conversion may be mediated by CCN2 (CTGF) (32). Thirdly, $\mathrm{CCN} 3(\mathrm{NOV})$ is functionally related to genes in which mutations are disproportionately highly observed in schizophrenia cases relative to controls (33). Finally, the protein discoidin domain receptor 1 (DDR1) has been proposed as a receptor for CCN3 (34); DDR1 is involved in myelination processes in the brain (35), and there is robust evidence for an association between genetic variants around the DDR1 locus and psychotic illness, possibly mediated via white matter abnormalities (36-39). Conceivably, therefore, any CCN3 effects on psychosis risk might be mediated via DDR1.

In addition to effects on psychosis risk, perturbed expression of CCN2 and/or CCN3 might impact upon mood symptoms and relevant personality traits. CCN2 (CTGF) has recently been implicated as a prodepressant molecule on the basis of human and animal model studies (40), while CCN3 hippocampal expression is decreased $\sim 1.7$-fold in the "chronic social defeat" mouse model of depression (41). Consistent with the results from the STS enzyme inhibition study, brain-specific deletion of Ccn3 has an anxiolytic effect in mice (42). New findings from a large Mendelian Randomization study have suggested that plasma CCN2 is significantly causally-associated with neuroticism and possibly also with depression $(\mathrm{p}=0.07)$, and that plasma CCN3 is significantly causally-associated with anxiety/panic attacks and agreeableness $(43,44)$; in bipolar disorder, neurotic and (negative) agreeableness traits are reliable predictors of depressive and manic symptoms, respectively (45).

Among some women with bipolar disorder, sleep deprivation appears to be a trigger for acute mood (manic) symptoms, and it is these individuals who appear to be at particularly high risk of developing PP (46). Emerging data hints that elevated blood CCN3 levels are associated with obstructive sleep apnea (OSA), a common condition associated with impaired sleep and subsequent cognitive processing, and with irritable or depressed mood (47). Feasibly, a predisposition to sleep disruption as a consequence of high CCN3 levels and heightened OSA risk may play a role in vulnerability to PP.
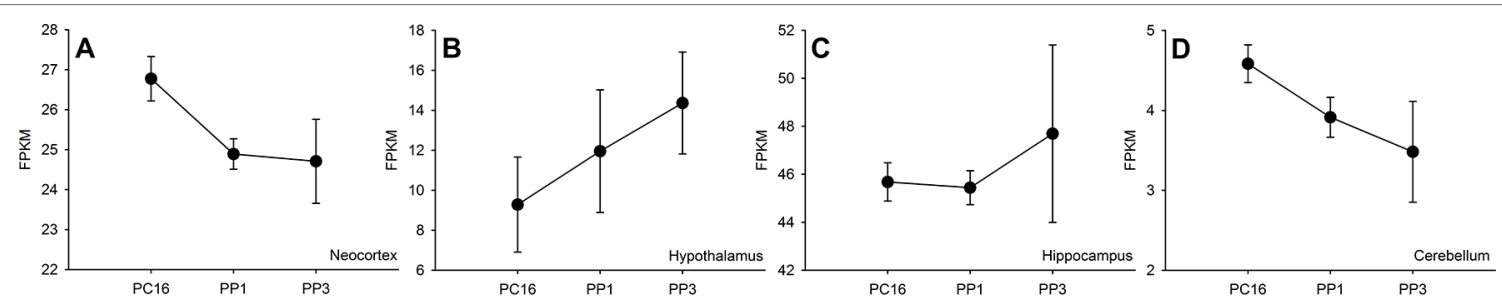

FIGURE 1 | Expression of Ccn3 across neocortex (A), hypothalamus (B), hippocampus (C) and cerebellum (D) regions of C57BL/6J mouse brain from mid-late pregnancy (postconception day 16, PC16) to postpartum days 1 and 3 (PP1 and PP3). RNA sequencing data are taken from Ray et al. (30) and expression is measured via Fragments Per Kilobase of transcript per Million mapped reads (FPKM) across three biological replicates per timepoint (mean and standard error of the mean shown for each timepoint). 


\section{Expression Regulated by Pro- and Anti- Psychotic Factors Relevant to PP}

There is evidence from both in vivo and in vitro studies that exposure to pro- and anti-psychotic factors can influence $\mathrm{CCN}$ family gene expression; however, many of these studies have focussed on tissues other than the brain, and the extent to which their results can be generalized to this tissue remains to be confirmed.

Of relevance to the idea that a precipitous drop in maternal circulating steroid hormones (notably oestrogens) may somehow elicit psychosis in the postpartum period, work in rats has demonstrated that ovariectomy, and the associated decrease in circulating oestrogens, results in significantly decreased Ccn3 (Nov) expression in the hippocampus (48). Chronic stress results in upregulation of the Ccn2 (Ctgf) gene in mouse hippocampus (49), while the administration of psychotomimetic or antipsychotic drugs results in increased CCN gene expression across the rodent brain $(50,51)$. In the STS inhibition mouse model described above, the elevation in brain Ccn3 expression could be alleviated by concurrent administration of the clinically-efficacious antipsychotic ziprasidone (18), while in rats, oral administration of lithium (a mood-stabilizing drug used to treat PP), is associated with substantial, and relatively specific, upregulation of Ccn3 kidney expression (52). In women with a history of bipolar disorder/psychosis, smoking appears to be associated with vulnerability to psychotic episodes during pregnancy and in the puerperium $(53,54)$; in female mouse lung tissue, extended exposure to cigarette smoke has been associated with downregulation of CCNs 1, 3 and 4 (55).

Finally, in vitro studies have shown that $\mathrm{CCN}$ expression can be finely regulated by a diverse range of inflammatory mediators including cytokines (TNFa, IL1 $\beta$, TGF- $\beta$ ), prostaglandins, nitric oxide, histamine, serotonin, and extracellular matrix enzymes in a tissue-specific manner (56).

\section{Role as a Nexus Between PP-Relevant Immune System and Neuroanatomical Measures}

Individuals with first-onset PP have been reported to show hyperactivation of the monocyte/macrophage arm of the immune system (57), possibly as a downstream consequence of changes in the abundance and/or activity of subsets of immunosuppressive regulatory T-cells (Tregs) $(57,58)$. The proportion of circulating Tregs also appears to be abnormal (generally lowered) in individuals with bipolar disorder (59-61).

Although little is currently known about the neuroanatomy of $\mathrm{PP}$, pilot data indicates that individuals at high risk of PP exhibit abnormal myelination in the temporal lobe, and in sublobar areas (62), and one case of PP presented with white matter abnormalities within the splenium of the corpus callosum (63). Interestingly, in patients with bipolar disorder, the frequency of circulating Tregs correlates positively with markers of white matter integrity (64). Together these results suggest: a) that defective myelination processes may predispose to PP and associated conditions, and b) the possibility of a biological link between immune function and myelination processes across these conditions.

Work by Dombrowki and colleagues has shown that CCN3 is secreted by Tregs, and mediates brain (re)myelination processes in mammals (12). On the basis of this finding, we have proposed that perturbations in the Treg-CCN3-(re)myelination axis may confer vulnerability to PP (65). The high expression of CCN3 in the temporal cortex, and adjacent structures, may potentially explain the myelination abnormalities seen here in patients with PP. Additionally, an in vitro study in mouse cortical neurons in which CCN3 was overexpressed showed impaired midline crossing of callosal projection neurons (66), providing a viable explanation for the corpus callosum structural abnormalities described in the PP case above.

\section{Putative Role in Conditions Comorbid With PP}

Convergent findings from large general population, and diseasespecific cohorts, support a robust association between the medical condition pre-eclampsia and postpartum psychiatric symptoms $(67,68)$. Pre-eclampsia is a potentially lifethreatening complication of late pregnancy, characterized by the onset of high blood pressure and proteinuria (69). Like PP, the pathophysiology of pre-eclampsia is poorly-understood, but abnormal placental invasion and vascularization processes are thought to be important (69); chronic maternal hypertension is a significant risk factor $(69,70)$.

Biological factors which have pleiotropic (independent) effects on both pre-eclampsia and postpartum mood disorder risk may explain the observed link between the two conditions. Alternatively, these factors may solely (or predominantly) influence pre-eclampsia risk, and the development of this condition may then precipitate symptoms associated with PP via mechanisms such as heightened perinatal stress or via adverse effects of hypertension on neural processes. CCN proteins, and $\mathrm{CCN} 3$ in particular, represent plausible pleiotropic biological risk factors.

$\mathrm{CCN}$ family members influence angiogenesis across a variety of tissues, and in the placenta CCN1 and CCN3 are expressed in the endothelial cells of placental vessels with expression increasing throughout pregnancy (71); in the placenta, CCNs1-3 seem to act as regulators of trophoblast proliferation and migration, with CCN2 acting antagonistically (71). CCN1 and CCN3 levels are decreased in both the placental tissue and serum of mothers with early-onset pre-eclampsia, whereas CCN2 levels are increased in the serum of women with severe pre-eclampsia (71). In addition to a possible role in pre-eclampsia pathophysiology, CCN3 may play a role in affecting blood pressure more generally; the gene is very highly expressed in the zona glomerulosa of the adrenal gland, a tissue involved in the regulation of blood pressure via aldosterone secretion (72). Consistent with this, genomic studies have identified a genetic polymorphism within CCN3 (rs2071518) associated with blood pressure regulation (73), and plasma CCN3 is highly-significantly causally-linked to blood pressure $(43,44)$. 
Given the well-established relationship between bipolar disorder and PP risk, one might expect $\mathrm{CCN}$ proteins to influence cellular processes relevant to both disorders. CCN3 is thought to regulate intracellular calcium levels (74), and aberrant calcium signaling has been implicated in both bipolar disorder (75) and PP (76) risk. Finally, CCN proteins can influence Notch and Wnt signaling cascades $(10,71)$, the dysfunction of which has been reported in both bipolar disorder and schizophrenia $(77,78)$ and in cases presenting with postpartum psychiatric symptoms (79).

\section{DISCUSSION}

Currently, the pathophysiology of PP is poorly-understood. The rarity and complexity of the condition precludes large casecontrol biochemical and genetic studies, and there is arguably a need to focus on candidate systems. Historically, however, candidate gene or system-led approaches have been associated with high rates of false positive and negative findings (80), though some of this poor reproducibility may be accounted for by the relatively arbitrary selection of candidates based upon limited and unreliable theory. Above, I have discussed why I believe the CCN family represents a strong candidate system warranting further investigation in PP; a putative pathophysiological model based upon these arguments is presented in Figure 2. Despite there being a strong relationship between a prior history of bipolar disorder and PP risk, to date, there is little evidence from genomic and physiological studies for abnormal CCN function in bipolar disorder; this "absence of evidence" may be due to technical limitations, or alternatively, as CCN gene/protein levels fluctuate considerably throughout pregnancy and the postpartum period, it is plausible that it is specifically abnormalities in the expression/function of these proteins (against a background of bipolar disorder-associated pathophysiological changes) that confer PP risk. Additionally, it should be appreciated that while a link between CCN proteins and PP is explicitly discussed here, the functions/attributes of the CCN family mean that these proteins could also feasibly play a role in disorders with overlapping symptoms and biology, notably postpartum depression and anxiety.

Future work may involve comparing the levels of CCN2 and $\mathrm{CCN} 3$ proteins (and their interactors) in peripheral tissues in

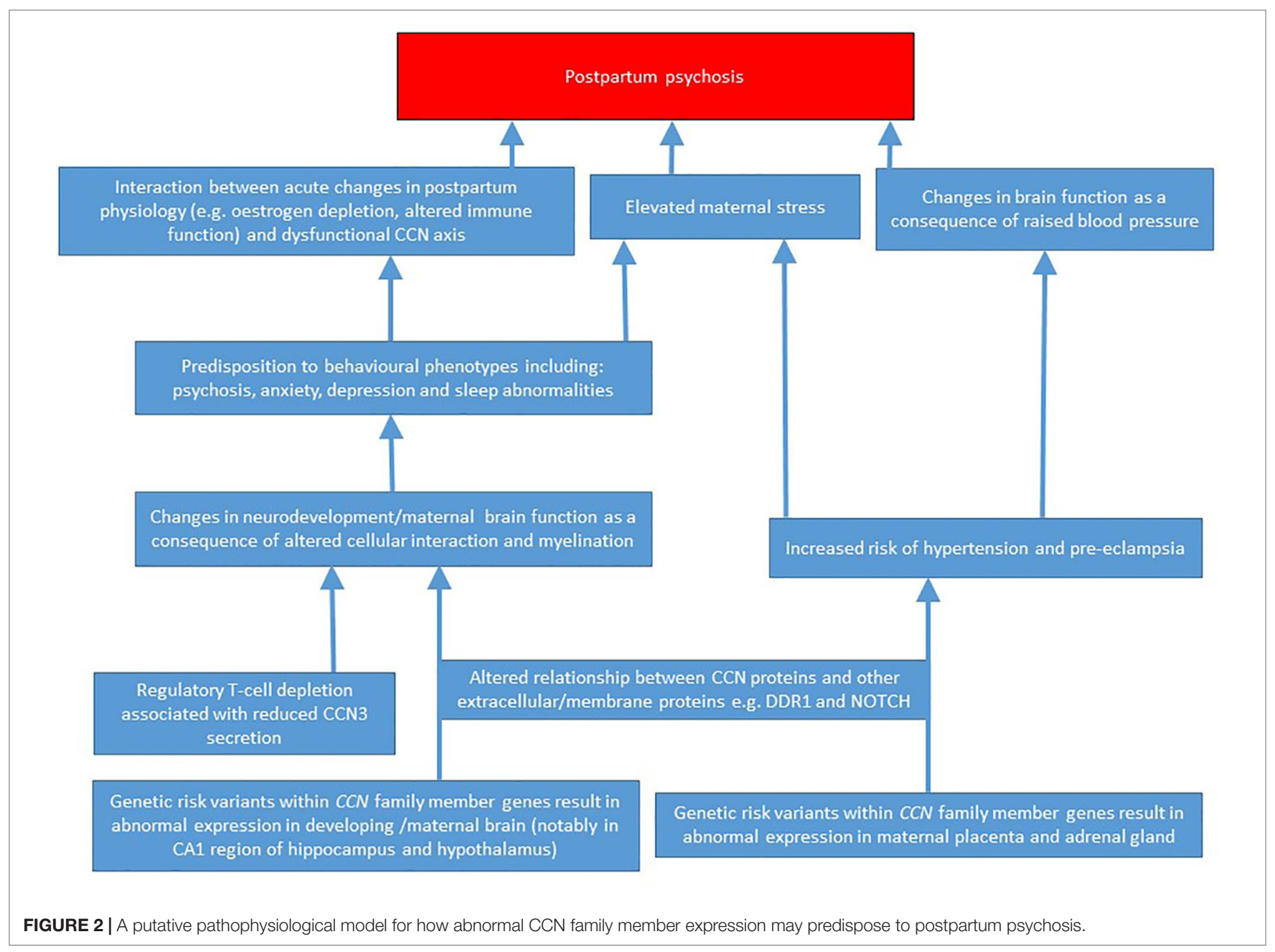


individuals with $\mathrm{PP}$, in individuals at risk of PP, and in healthy controls across pregnancy and the postpartum period, as well as comparing the sequences of these genes across groups. Parallel animal model studies, in which levels of CCN2 and CCN3 proteins are systematically varied and PP-relevant neurobiological and behavioral measures assessed, may be useful for indicating causality.

Should the work above suggest that changes in the CCN family are associated with, or causal for, an increased risk of postpartum psychopathology, they could act as predictive biomarkers, and may be amenable to normalization through therapeutic approaches currently under development (81).

\section{REFERENCES}

1. VanderKruik R, Barreix M, Chou D, Allen T, Say L, Cohen LS, et al. The global prevalence of postpartum psychosis: a systematic review. BMC Psychiatry (2017) 17:272. doi: 10.1186/s12888-017-1427-7

2. Bergink V, Rasgon N, Wisner KL. Postpartum Psychosis: Madness, Mania, and Melancholia in Motherhood. Am J Psychiatry (2016) 173:1179-88. doi: 10.1176/appi.ajp.2016.16040454

3. Brockington I. Suicide and filicide in postpartum psychosis. Arch Womens Ment. Health (2017) 20:63-9. doi: 10.1007/s00737-016-0675-8

4. Poels EMP, Bijma HH, Galbally M, Bergink V. Lithium during pregnancy and after delivery: a review. Int J Bipolar Disord (2018) 6:26. doi: 10.1186/ s40345-018-0135-7

5. Davies W. Understanding the pathophysiology of postpartum psychosis: Challenges and new approaches. World J Psychiatry (2017) 7:77-88. doi: 10.5498/wjp.v7.i2.77

6. Neale BM, Sklar P. Genetic analysis of schizophrenia and bipolar disorder reveals polygenicity but also suggests new directions for molecular interrogation. Curr Opin Neurobiol (2015) 30: 131-8. doi: 10.1016/j. conb.2014.12.001

7. Jones I, Chandra PS, Dazzan P, Howard LM. Bipolar disorder, affective psychosis, and schizophrenia in pregnancy and the post-partum period. Lancet (2014) 384: 1789-99. doi: 10.1016/S0140-6736(14)61278-2

8. Takigawa M. The CCN Proteins: an overview. Methods Mol Biol (2017) 1489:1-8. doi: 10.1007/978-1-4939-6430-7_1

9. Lubbers BR, Smit AB, Spijker S, van den Oever MC. Neural ECM in addiction, schizophrenia, and mood disorder. Prog Brain Res (2014) 214: 263-84. doi: 10.1016/B978-0-444-63486-3.00012-8

10. Perbal B. The concept of the CCN protein family revisited: a centralized coordination network. J Cell Commun Signal (2018) 12:3-12. doi: 10.1007/ s12079-018-0455-5

11. Hoshijima M, Hattori T, Aoyama E, Nishida T, Yamashiro T, Takigawa M. Roles of heterotypic CCN2/CTGF-CCN3/NOV and homotypic CCN2-CCN2 interactions in expression of the differentiated phenotype of chondrocytes. FEBS J (2012) 279:3584-97. doi: 10.1111/j.1742-4658.2012.08717.x

12. Dombrowski Y, O’Hagan T, Dittmer M, Penalva R, Mayoral SR, Bankhead P, et al. Regulatory $\mathrm{T}$ cells promote myelin regeneration in the central nervous system. Nat Neurosci (2017) 20:674-80. doi: 10.1038/nn.4528

13. Ercan E, Han JM, Di Nardo A, Winden K, Han MJ, Hoyo L, et al. Neuronal CTGF/CCN2 negatively regulates myelination in a mouse model of tuberous sclerosis complex. J Exp Med (2017) 214:681-97. doi: 10.1084/jem.20160446

14. Lamond R, Barnett SC. Schwann cells but not olfactory ensheathing cells inhibit CNS myelination via the secretion of connective tissue growth factor. J Neurosci (2013) 33: 18686-97. doi: 10.1523/JNEUROSCI.3233-13.2013

15. Curran HV, D'Souza DC, Robbins TW, Fletcher P. Modelling psychosis. Psychopharmacol (Berl.) (2009) 206: 513-4. doi: 10.1007/s00213-009-1663-8

16. Cavenagh A, Chatterjee S, Davies W. Behavioural and psychiatric phenotypes in female carriers of genetic mutations associated with X-linked ichthyosis. PloS One (2019) 14:e0212330. doi: 10.1371/journal.pone.0212330

17. Davies W. Does steroid sulfatase deficiency influence postpartum psychosis risk? Trends Mol Med (2012) 18: 256-62. doi: 10.1016/j.molmed.2012.03.001

\section{AUTHOR CONTRIBUTIONS}

The author confirms being the sole contributor of this work and has approved it for publication.

\section{FUNDING}

The work was supported by Medical Research Council (MRC) United Kingdom Centre Grant MR/L010305/1 (https://mrc. ukri.org/) and Cardiff University. The funders had no role in study design, data collection and analysis, decision to publish, or preparation of the manuscript.

18. Humby T, Cross ES, Messer L, Guerrero S, Davies W. A pharmacological mouse model suggests a novel risk pathway for postpartum psychosis. Psychoneuroendocrinology (2016) 74:363-70. doi: 10.1016/j.psyneuen. 2016.09.019

19. Creeth HDJ, McNamara GI, Tunster SJ, Boque-Sastre R, Allen B, Sumption L, et al. Maternal care boosted by paternal imprinting in mammals. PloS Biol (2018) 16:e2006599. doi: 10.1371/journal.pbio.2006599

20. Quilter CR, Blott SC, Wilson AE, Bagga MR, Sargent CA, Oliver GL, et al. Porcine maternal infanticide as a model for puerperal psychosis. Am J Med Genet B. Neuropsychiatr Genet (2007) 144B: 862-8. doi: 10.1002/ ajmg.b.30529

21. Landers CA. (2019). "Using a Porcine Maternal Infanticide Model to Investigate the Inheritance of Human Puerperal Psychosis," Ph.D thesis, University of Cambridge, UK. doi: 10.17863/CAM.31731

22. Quilter CR, Sargent CA, Bauer J, Bagga MR, Reiter CP, Hutchinson EL, et al. An association and haplotype analysis of porcine maternal infanticide: a model for human puerperal psychosis? Am J Med Genet B Neuropsychiatr Genet (2012) 159B: 908-27. doi: 10.1002/ajmg.b.32097

23. Zhang Y, Wang C. Nephroblastoma overexpressed (NOV/CCN3) gene: a paired-domain-specific PAX3-FKHR transcription target that promotes survival and motility in alveolar rhabdomyosarcoma cells. Oncogene (2011) 30: 3549-62. doi: 10.1038/onc.2011.69

24. Jones I, Hamshere M, Nangle JM, Bennett P, Green E, Heron J, et al. Bipolar affective puerperal psychosis: genome-wide significant evidence for linkage to chromosome 16. Am J Psychiatry (2007) 164: 1099-104. doi: 10.1176/ ajp.2007.164.7.1099

25. Malik AR, Liszewska E, Jaworski J. Matricellular proteins of the Cyr61/ CTGF/NOV (CCN) family and the nervous system. Front Cell Neurosci (2015) 9:237. doi: 10.3389/fncel.2015.00237

26. Braineac database (www.braineac.org) (Accessed May 14, 2019).

27. Allen Brain Atlas (www.brain-map.org) (Accessed May 14, 2019).

28. Fuste M, Pauls A, Worker A, Reinders A, Simmons A, Williams SCR, et al. Brain structure in women at risk of postpartum psychosis: an MRI study. Transl Psychiatry (2017) 7:1286. doi: 10.1038/s41398-017-0003-8

29. Haukvik UK, Tamnes CK, Soderman E, Agartz I. Neuroimaging hippocampal subfields in schizophrenia and bipolar disorder: a systematic review and meta-analysis. J Psychiatr Res (2018) 104:217-26. doi: 10.1016/j. jpsychires.2018.08.012

30. Ray S, Tzeng RY, DiCarlo LM, Bundy JL, Vied C, Tyson G, et al. An examination of dynamic gene expression changes in the mouse brain during pregnancy and the postpartum period. G3. (Bethesda) (2015) 6: 221-33. doi: $10.1534 / \mathrm{g} 3.115 .020982$

31. Enwright Iii JF, Huo Z, Arion D, Corradi JP, Tseng G, Lewis DA. Transcriptome alterations of prefrontal cortical parvalbumin neurons in schizophrenia. Mol Psychiatry (2018) 23:1606-13. doi: 10.1038/ mp.2017.216

32. Jeffries CD, Perkins DO, Fournier M, Do KQ, Cuenod M, Khadimallah I, et al. Networks of blood proteins in the neuroimmunology of schizophrenia. Transl Psychiatry (2018) 8:112. doi: 10.1038/s41398-018-0158-y

33. Fulgitini V. (2018). "Testing the network hypothesis for schizophrenia and autism spectrum disorder using whole exome sequencing data" Ph.D 
thesis, McGill University, Canada http://digitool.library.mcgill.ca/webclient/ StreamGate?folder_id $=0 \&$ dvs $=1557839982491 \sim 816$.

34. Fukunaga-Kalabis M, Martinez G, Liu ZJ, Kalabis J, Mrass P, Weninger W, et al. CCN3 controls 3D spatial localization of melanocytes in the human skin through DDR1. J Cell Biol (2006) 175: 563-9. doi: 10.1083/jcb.200602132

35. Roig B, Franco-Pons N, Martorell L, Tomas J, Vogel WF, Vilella E. Expression of the tyrosine kinase discoidin domain receptor 1 (DDR1) in human central nervous system myelin. Brain Res (2010) 1336: 22-9. doi: 10.1016/j. brainres.2010.03.099

36. Gas C, Canales-Rodriguez EJ, Radua J, Abasolo N, Cortes MJ, Salvado E, et al. Discoidin domain receptor 1 gene variants are associated with decreased white matter fractional anisotropy and decreased processing speed in schizophrenia. J Psychiatr Res (2019) 110:74-82. doi: 10.1016/j.jpsychires.2018.12.021

37. Li Z, Chen J, Yu H, He L, Xu Y, Zhang D, et al. Genome-wide association analysis identifies 30 new susceptibility loci for schizophrenia. Nat Genet (2017) 49:1576-83. doi: 10.1038/ng.3973

38. C. Autism Spectrum Disorders Working Group of The Psychiatric Genomics. Meta-analysis of GWAS of over 16,000 individuals with autism spectrum disorder highlights a novel locus at 10q24.32 and a significant overlap with schizophrenia. Mol Autism (2017) 8:21. doi: 10.1186/s13229-017-0137-9

39. Roig B, Virgos C, Franco N, Martorell L, Valero J, Costas J, et al. The discoidin domain receptor 1 as a novel susceptibility gene for schizophrenia. Mol Psychiatry (2007) 12: 833-41. doi: 10.1038/sj.mp.4001995

40. Turner CA, Sharma V, Hagenauer MH, Chaudhury S, O'Connor AM, HebdaBauer EK, et al. Connective Tissue Growth Factor Is a Novel Prodepressant. Biol Psychiatry (2018) 84:555-62. doi: 10.1016/j.biopsych.2018.04.013

41. Azzinnari D. (2014). "Towards increased understanding of stress-related depression and its treatment: neurobehavioural studies in mice," Ph.D thesis. Switzerland: University of Zurich. https://www.zora.uzh.ch/id/ eprint/112898/1/PhD\%20Thesis\%20of\%20Damiano\%20Azzinnari.pdf.

42. Jackson Laboratory website (https://www.jax.org/strain/028614) (Accessed May 14, 2019).

43. Zheng J, Haberland V, Baird D, Walker V, P. haycock A, Richardson TG, et al. Phenome-wide Mendelian randomisation mapping the influence of the plasma proteome on complex diseases. bioRxiv (2019). doi: 10.1101/627398.

44. EpiGraphDB (http://www.epigraphdb.org/pqtl/) (Accessed May 14, 2019).

45. Quilty LC, Sellbom M, Tackett JL, Bagby RM. Personality trait predictors of bipolar disorder symptoms. Psychiatry Res (2009) 169: 159-63. doi: 10.1016/j.psychres.2008.07.004

46. Lewis KJS, Di Florio A, Forty L, Gordon-Smith K, Perry A, Craddock N, et al. Mania triggered by sleep loss and risk of postpartum psychosis in women with bipolar disorder. J Affect Disord (2018) 225:624-9. doi: 10.1016/j. jad.2017.08.054

47. Weingarten JA, Bellner L, Peterson SJ, Zaw M, Chadha P, Singh SP, et al. The association of NOV/CCN3 with obstructive sleep apnea (OSA): preliminary evidence of a novel biomarker in OSA. Horm Mol Biol Clin Invest (2017) 31. doi: 10.1515/hmbci-2017-0029

48. Savari M, Kallo I, Hrabovszky E, Solymosi N, Liposits Z. Ovariectomy alters gene expression of the hippocampal formation in middle-aged rats. Endocrinology (2017) 158:69-83. doi: 10.1210/en.2017-00514

49. Stankiewicz AM, Goscik J, Majewska A, Swiergiel AH, Juszczak GR. The effect of acute and chronic social stress on the hippocampal transcriptome in mice. PloS One (2015) 10:e0142195. doi: 10.1371/journal.pone.0142195

50. Sakuma K, Komatsu H, Maruyama M, Imaichi S, Habata Y, Mori M. Temporal and spatial transcriptional fingerprints by antipsychotic or propsychotic drugs in mouse brain. PloS One (2015) 10:e0118510. doi: 10.1371/journal.pone.0118510

51. Ito T, Hiraoka S, Kuroda Y, Ishii S, Umino A, Kashiwa A, et al. Effects of schizophrenomimetics on the expression of the CCN1 (CYR 61) gene encoding a matricellular protein in the infant and adult neocortex of the mouse and rat. Int J Neuropsychopharmacol (2007) 10: 717-25. doi: 10.1017/ S1461145707007882

52. Marti HP, Jeffs A, Scherer A, Leader J, Leader C, Bedford J, et al. Renal Fibrosis mRNA Classifier: Validation in Experimental Lithium-Induced Interstitial Fibrosis in the Rat Kidney. PloS One (2016) 11:e0168240. doi: 10.1371/journal.pone.0168240

53. Taylor CL, Broadbent M, Khondoker M, Stewart RJ, Howard LM. Predictors of severe relapse in pregnant women with psychotic or bipolar disorders. J Psychiatr Res (2018) 104:100-7. doi: 10.1016/j.jpsychires.2018.06.019
54. Di Florio A, Morgan H, Jones L, Forty L, Gordon-Smith K, Craddock N, et al. Smoking and postpartum psychosis. Bipolar Disord (2015) 17: 572-3. doi: 10.1111/bdi.12314

55. Gueugnon F, Thibault VC, Kearley J, Petit-Courty A, Vallet A, Guillon A, et al. Altered expression of the $\mathrm{CCN}$ genes in the lungs of mice in response to cigarette smoke exposure and viral and bacterial infections. Gene (2016) 586: 176-83. doi: 10.1016/j.gene.2016.04.022

56. Kular L, Pakradouni J, Kitabgi P, Laurent M, Martinerie C. The CCN family: a new class of inflammation modulators? Biochimie (2011) 93: 377-88. doi: 10.1016/j.biochi.2010.11.010

57. Bergink V, Burgerhout KM, Weigelt K, Pop VJ, de Wit H, Drexhage RC, et al. Immune system dysregulation in first-onset postpartum psychosis. Biol Psychiatry (2013) 73: 1000-7. doi: 10.1016/j.biopsych.2012.11.006

58. Kumar MM, Venkataswamy MM, Sathyanarayanan G, Thippeswamy H, Chandra PS, Mani RS. Immune system aberrations in postpartum psychosis: an immunophenotyping study from a tertiary care neuropsychiatric hospital in India. J Neuroimmunol. (2017) 310:8-13. doi: 10.1016/j. jneuroim.2017.06.002

59. Vogels RJ, Koenders MA, van Rossum EF, Spijker AT, Drexhage HA. $\mathrm{T}$ cell deficits and overexpression of hepatocyte growth factor in antiinflammatory circulating monocytes of middle-aged patients with bipolar disorder characterized by a high prevalence of the metabolic syndrome. Front Psychiatry (2017) 8:34. doi: 10.3389/fpsyt.2017.00034

60. Barbosa IG, Rocha NP, Assis F, Vieira EL, Soares JC, Bauer ME, et al. Monocyte and lymphocyte activation in bipolar disorder: a new piece in the puzzle of immune dysfunction in mood disorders. Int J Neuropsychopharmacol (2014) 18, 1-7. doi: 10.1093/ijnp/pyu021

61. do Prado CH, Rizzo LB, Wieck A, Lopes RP, Teixeira AL, GrassiOliveira R, et al. Reduced regulatory $\mathrm{T}$ cells are associated with higher levels of Th1/TH17 cytokines and activated MAPK in type 1 bipolar disorder. Psychoneuroendocrinology (2013) 38: 667-76. doi: 10.1016/j. psyneuen.2012.08.005

62. Giordano A, Pauls AM, Hazelgrove K, Combes A, Wood T, Mehta MA, et al. Neuroinflammation in puerperal psychosis: the relationship between myelin content and peripheral inflammatory markers. Schizophr Bull (2017) 43(S1):S144. doi: 10.1093/schbul/sbx023.084

63. Udaya SC, Chauhan BN, Philip VJ. Bright splenium of a psychotic mind. Ann Indian Acad Neurol (2015) 18: 80-3. doi: 10.4103/0972-2327.145287

64. Poletti S, de Wit H, Mazza E, Wijkhuijs AJM, Locatelli C, Aggio V, et al. Th17 cells correlate positively to the structural and functional integrity of the brain in bipolar depression and healthy controls. Brain Behav Immun (2017) 61:317-25. doi: 10.1016/j.bbi.2016.12.020

65. Dazzan P, Fuste M, Davies W. Do Defective Immune System-Mediated Myelination Processes Increase Postpartum Psychosis Risk? Trends Mol Med (2018) 24:942-9. doi: 10.1016/j.molmed.2018.09.002

66. Park M, Baek IJ, Kim H, Woo DK, Park YJ, Shim S. CCN3 overexpression inhibits growth of callosal projections via upregulation of RAB25. Biochem Biophys Res Commun (2015) 461: 456-62. doi: 10.1016/j. bbrc.2015.04.016

67. Meltzer-Brody S, Howard LM, Bergink V, Vigod S, Jones I, Munk-Olsen T, et al. Postpartum psychiatric disorders. Nat Rev Dis Primers (2018) 4:18022. doi: 10.1038/nrdp.2018.22

68. Bergink V, Laursen TM, Johannsen BM, Kushner SA, Meltzer-Brody S, Munk-Olsen T. Pre-eclampsia and first-onset postpartum psychiatric episodes: a Danish population-based cohort study. Psychol Med (2015) 45: 3481-9. doi: 10.1017/S0033291715001385

69. Phipps EA, Thadhani R, Benzing T, Karumanchi SA. Pre-eclampsia: pathogenesis, novel diagnostics and therapies. Nat Rev Nephrol (2019) 15:275-89. doi: 10.1038/s41581-019-0119-6

70. Bartsch E, Medcalf KE, Park AL, Ray JGand G. High Risk of Pre-eclampsia Identification. Clinical risk factors for pre-eclampsia determined in early pregnancy: systematic review and meta-analysis of large cohort studies. BMJ (2016) 353:i1753. doi: 10.1136/bmj.i1753

71. Winterhager E, Gellhaus A. The role of the CCN family of proteins in female reproduction. Cell Mol Life Sci (2014) 71: 2299-311. doi: 10.1007/ s00018-014-1556-9

72. Bergman J, Botling J, Fagerberg L, Hallstrom BM, Djureinovic D, Uhlen M, et al. The human adrenal gland proteome defined by transcriptomics and 
antibody-based profiling. Endocrinology (2017) 158:239-51. doi: 10.1210/ en.2016-1758

73. Wain LV, Vaez A, Jansen R, Joehanes R, Erzurumluoglu AM, O'Reilly PF, et al. Novel blood pressure locus and gene discovery using genome-wide association study and expression data sets from blood and the kidney. Hypertension (2017). 70:e4-e19 doi: 10.1161/HYPERTENSIONAHA. 117.09438

74. Lombet A, Planque N, Bleau AM, Li CL, Perbal B. CCN3 and calcium signaling. Cell Commun Signal (2003) 1:1. doi: 10.1186/1478-811X-1-1

75. Kato T. Current understanding of bipolar disorder: toward integration of biological basis and treatment strategies. Psychiatry Clin Neurosci (2019) 73:526-540. doi: 10.1111/pcn.12852

76. Riley DM, Watt DC. Hypercalcemia in the etiology of puerperal psychosis. Biol Psychiatry (1985) 20: 479-88. doi: 10.1016/0006-3223(85)90020-4

77. Hoseth EZ, Krull F, Dieset I, Morch RH, Hope S, Gardsjord ES, et al. Attenuated Notch signaling in schizophrenia and bipolar disorder. Sci Rep (2018) 8:5349. doi: 10.1038/s41598-018-23703-w

78. Hoseth EZ, Krull F, Dieset I, Morch RH, Hope S, Gardsjord ES, et al. Exploring the Wnt signaling pathway in schizophrenia and bipolar disorder. Transl Psychiatry (2018) 8:55. doi: 10.1038/s41398-018-0102-1
79. Pantoni L, Pescini F, Inzitari D, Dotti MT. Postpartum psychiatric disturbances as an unrecognized onset of CADASIL. Acta Psychiatr Scand (2005) 112:241. doi: 10.1111/j.1600-0447.2005.00595.x author reply 242.

80. Duncan LE, Ostacher M, Ballon J. How genome-wide association studies (GWAS) made traditional candidate gene studies obsolete. Neuropsychopharmacology (2019) 44:1518-1523. doi: 10.1038/s41386-019-0389-5

81. Jun JI, Lau LF. Taking aim at the extracellular matrix: CCN proteins as emerging therapeutic targets. Nat Rev Drug Discovery (2011) 10: 945-63. doi: $10.1038 / \mathrm{nrd} 3599$

Conflict of Interest: The author declares that the research was conducted in the absence of any commercial or financial relationships that could be construed as a potential conflict of interest.

Copyright (๑) 2019 Davies. This is an open-access article distributed under the terms of the Creative Commons Attribution License (CC BY). The use, distribution or reproduction in other forums is permitted, provided the original author(s) and the copyright owner(s) are credited and that the original publication in this journal is cited, in accordance with accepted academic practice. No use, distribution or reproduction is permitted which does not comply with these terms. 